\title{
BLADDER-SPHINCTER DYSFUNCTION IN CHILDHOOD
}

\author{
STEPHEN A. KOFF, M.D. \\ From the Pediatric Urology Service, C. S. Mott Children's Hospital, \\ and the Section of Urology, Department of Surgery, University of \\ Michigan Medical Center, Ann Arbor, Michigan
}

\begin{abstract}
While it is obvious that the combination of elevated intravesical pressure, urinary tract infection, and reflux can be devastating to the upper urinary tracts, it has been appreciated only recently that a single mechanism, obstruction due to disorders of vesicourethral function, can produce all three pathologic phenomena in neurologically normal children. Advances in pediatric urodynamics now permit these patients to be recognized and their particular pattern of bladder and sphincter dysfunction to be diagnosed with certainty. Individualized therapy aimed at the underlying functional disturbance is often successful and may make surgical therapy unnecessary.
\end{abstract}

The idea that urinary tract infection, obstruction, and vesicoureteral reflux can be manifestations of disordered function of the lower urinary tract in normal children has been slow to evolve. This notion appears to upset traditional urologic principles which focus on anatomic and bacteriologic causes for urinary tract dysfunction. Consequently, in the absence of structural obstruction, the child with urinary tract infection and reflux has been considered usually to have an idiopathic disease. Recent developments in the field of urodynamics, however, support this concept by demonstrating that obstructing urinary tract lesions may be caused by incoordination between the bladder and the urinary sphincter and may be as severe and as pathophysiologically significant as those produced by anatomic obstruction. Widespread use of newer urodynamic techniques has permitted an accurate definition of these functional voiding disturbances and has documented their frequent occurrence in neurologically normal children.

The most striking feature of significant voiding disturbances is that they begin to develop at about the time of toilet training and appear to represent aberrant or improperly learned patterns of urinary control. Recognition of this relationship to toilet training and appreciation of the significance of dysfunctional voiding has had an increasing impact on the over-all management of childhood urinary tract disease. In some cases, unidentified and untreated voiding dysfunction has been shown to be responsible for failure of surgery to correct reflux while in many instances treatment of underlying vesicourethral disturbance has eliminated recurrent infection and allowed reflux to disappear spontaneously. Consequently, physicians who care for children with urinary tract infection and reflux must be aware of these pathologic disturbances of bladder and sphincter function which produce obstruction and must be able to diagnose and treat them adequately.

In the following discussion we will examine the important syndromes of disordered lower urinary tract function occurring in normal children after first reviewing the development of normal bladder and sphincter control.

\section{Development of Normal Urinary Control}

In the infant, micturition occurs as a simple spinal cord reflex. When increasing amounts of bladder urine sufficiently stimulate the afferent limb of the reflex arc, contraction of the detrusor occurs. Even in the infant, the periurethral striated muscles which comprise the voluntary (external) urinary sphincter are fully 
integrated into the voiding reflex. As the bladder fills, the urinary sphincter constricts progressively to prevent incontinence. During micturition, reflex relaxation of the sphincter occurs simultaneously to permit low-pressure bladder emptying.

As the child matures, toilet training success and the achievement of urinary control depend on the outcome of three separate events in the development of bladder form and function. ${ }^{1}$ First, the capacity of the bladder must increase to permit it to function as a reservoir. Second, voluntary control over the periurethral striated muscle sphincter must occur to allow decisive initiation and termination of micturition. As with other acquired striated muscle skills, sphincter control fits into the orderly scheme of developmental landmarks and usually occurs by the age of three years. Third, direct cortical control over the spinal cord micturition reflex must develop in order for the child voluntarily to initiate or inhibit detrusor contraction. This last step in the development of urinary control is probably the most complicated, but once accomplished, the ability voluntarily to void or inhibit voiding at any degree of bladder filling sets the human child apart from all other mammals (except the canine). ${ }^{2}$

Eventually, by the age of four years, most children are toilet trained and have developed the adult pattern of urinary control. During filling of the bladder, this pattern is characterized by an absence of uninhibited or infantile detrusor contractions. Urodynamic studies confirm that even at bladder capacity, when the desire to void is strong, no detrusor contraction will occur unless initiated voluntarily. With reflex constriction of the urinary sphincter muscles occurring progressively during bladder filling, their contraction will become maximal at bladder capacity. When detrusor contraction is voluntarily started, simultaneous reflex relaxation of the sphincter permits low-pressure bladder emptying and normal urinary flow rates. ${ }^{3}$

\section{Syndromes of Detrusor-Sphincter Dysfunction}

In neurologically normal children the disorders of vesicourethral function which are significant enough to cause urinary tract infection, reflux, and urologic disease appear to represent abnormalities of toilet training and aberrations in the development of normal urinary control. ${ }^{4-7}$ It is likely that all children in making the transition from infantile to adult patterns of urinary control transiently display abnormalities of bladder and sphincter function. Provided that these are neither sustained nor repetitive they do not appear to be of any long-term consequence. However, those disturbances of bladder-sphincter function which are identified and persist after attempted toilet training generally increase in pathologic significance as the child becomes older. For purposes of discussion these disorders can be subdivided according to whether the dysfunction occurs during bladder filling or during bladder emptying.

\section{Detrusor-sphincter dysfunction during bladder filling}

The infantile, unstable, or so-called uninhibited bladder is a pattern of urinary dysfunction which is so common in childhood that often it is not considered an abnormality at all. Indeed, since uncontrolled contractions are the normal mechanism for infant micturition, it is likewise unclear how and when they become pathologic. Considered as an isolated event, the dysfunction caused by these contractions will never appear to be more of a problem than enuresis or incontinence; their pathophysiologic significance, however, becomes obvious when examined in the context of toilet training and learned voiding control.

Uninhibited bladder contractions are involuntary and unsuppressible detrusor contractions which occur in children who have failed to gain complete voluntary control over the micturition reflex. ${ }^{4,7-10}$ Their causation is unknown. They appear to represent either a delay in central nervous system maturation or a developmental regression with persistence of the infantile pattern of spontaneous bladder emptying. ${ }^{1} \mathrm{Al}$ though the uncontrolled contractions do not normally occur after the age of toilet training, they do not represent a neuropathy, and neurologic disease is rarely identified. Were it not for the fact that toilet-trained children have become physiologically able to constrict the urinary sphincter and have learned the social virtues of being continent, the uninhibited bladder would probably be of no clinical or pathologic significance.

The pathologic significance of uninhibited contractions depends entirely on the child's voluntary response to them which can be assessed using modern urodynamic techniques. Because uninhibited contractions are involuntary and unsuppressible, the child attempting to maintain continence during such contractions must voluntarily and tightly constrict the urinary 
sphincter to stay dry. This results in a simultaneous and unphysiologic contraction of both bladder and sphincter which has been termed detrusor-sphincter dyssynergia. During the involuntary detrusor contraction, high intravesical pressures develop and persist until either the bladder relaxes or empties. If, on the other hand, the child wishes to void during the uninhibited contraction, micturition will proceed normally but at low pressures to completion, because the urinary sphincter will reflexly relax during bladder contraction without volitional interference.

Voluntary constriction of the urinary sphincter during uninhibited bladder contractions has been shown to produce urinary obstruction and high intravesical pressure which is pathophysiologically identical to anatomic obstruction and to the bladder-sphincter incoordination seen in true neurogenic bladder disease. ${ }^{4,11}$ The signs and symptoms in each patient will vary and depend on the frequency and forcefulness of the contractions and on the effectiveness of sphincteric constriction. Reports indicate that the typical features of bladder hyperactivity (urgency, frequency, and precipitate micturition) occur in about 60 to 70 per cent of patients. ${ }^{4,7}$ Interestingly, up to one third of children have no incontinence, apparently due to their ability to overcome bladder contraction by tight sphincter constriction - unfortunately, at the expense of raised intravesical pressure. Characteristically, children with uninhibited bladders empty to completion without residue, and the occurrence of large residual urine volumes should make the diagnosis suspect. One sign which has been observed so frequently in little girls that it is almost pathognomonic of the condition is the Vincent curtsy, so named because the child squats and with the heel of one foot compresses the perineum and urethra to prevent urinary leakage. ${ }^{12}$

Although the precise incidence is unknown, urinary tract infections occur frequently in children with uninhibited bladders and are believed to be due to the repeatedly high intravesical pressure elevations, ${ }^{4,9-11}$ The distress in these patients may be heightened by the sensitizing effect of infection on bladder mucosal sensory neurons which increases bladder hyperactivity, produces pain, and may lead to spasm of the striated muscle sphincter. ${ }^{11,13-15}$

In addition to causing infection, repetitive obstruction and raised bladder pressures have been reported to produce a spectrum of in- travesical anatomic distortions in children with uninhibited contractions. Trabeculation, saccules, diverticula, and abnormalities of the ureteral orifices have been regularly observed in the absence of anatomic obstruction, and once acquired these changes tend to persist long after the symptoms of bladder dysfunction are eradicated. ${ }^{8}$ The occurrence of vesicoureteric reflux in up to 50 per cent of children with uninhibited bladders indicates the magnitude of the intravesical disturbance. ${ }^{4,9}$ The development and perpetuation of intravesical structural alterations and reflux in children with uninhibited bladders is analogous to the situation which occurs in true neuropathic bladder disease, the only difference being that sphincter uncoordination in the uninhibited bladder is induced voluntarily.

The basic approach to treating the uninhibited bladder in childhood is predicated on the observation that with maturation children will outgrow the tendency toward uncontrolled contractions and will develop the adult pattern of urinary control., ${ }^{4,16}$ Until such occurs, the goal of therapy is elimination of the uninhibited contractions without interfering with normal micturition. Anticholinergic drugs are the mainstay of treatment and are used to reduce detrusor hyperactivity, to increase the threshold volume at which uninhibited contractions occur, and to enlarge the functional capacity of the bladder. Our own preference is oxybutynin, which because of its long time of activity (six to eight hours) tends to provide smoother bladder control. High doses are often required (5 mg. tid) and must be titrated against anticholinergic drug side effects (flushing, dry mouth). ${ }^{17}$ In addition fluid restriction and frequent voidings are helpful therapeutic adjuncts to keep bladder volumes low.

The program combining anticholinergic drugs, fluid restriction, and frequent bladder emptyings has been shown effective in treating patients with uninhibited bladder contractions. Symptoms can usually be eliminated in over 80 per cent of children. ${ }^{4,7,9}$ Of interest in supporting the etiologic relationship between functional obstruction and organic urinary tract disease are reports which have demonstrated that nearly 60 per cent of children with uninhibited bladders and recurrent urinary tract infection can be maintained free of infection without antimicrobials by successful treatment of the uninhibited contractions alone. ${ }^{4,9}$ In contrast, the structural abnormalities such as diverticula and ureteral orifice changes with reflux do not 
appear to respond immediately to improved bladder control, although their precise resolution rates are unknown.

\section{Detrusor-sphincter dysfunction during bladder emptying}

If obstruction to urinary outflow occurs during voiding as a result of disordered bladder and sphincter function, urinary tract infection and intravesical structural abnormalities can occur. Collectively these functional disturbances have been called detrusor-sphincter dyssynergia, recognizing the pathologic state of simultaneous bladder and sphincter contraction during attempted micturition. This phenomenon is common and well recognized in patients with neurogenic bladder disease in whom altered neural transmissions cause the sphincter to constrict involuntarily during bladder contraction. In neurologically normal children, detrusorsphincter dyssynergia has been described in cases in which the incoordination or misuse of sphincter constriction was voluntary, as a consequence of inproperly learned patterns of urinary control.

The syndrome of the non-neurogenic, neurogenic bladder, or occult neuropathic bladder represents the severest form of dysfunctional voiding in childhood. ${ }^{18-22}$ The obstructive uropathy often seen in these patients has been interpreted by some investigators to indicate a true but undiagnosable bladder neuropathy. ${ }^{23}$ Although genuine controversy exists as to precise origin, Hinman ${ }^{18,19,21}$ and Allen ${ }^{5,22,24,25}$ have demonstrated that many patients appear to have acquired or learned abnormal voiding habits manifested as detrusor-sphincter dyssynergia. That this can be corrected or improved by noninvasive measures such as bladder retraining is convincing evidence for a functional rather than a neurologic origin.

The clinical features of this syndrome include incontinence that is often chronic and diurnal, fecal retention and soiling, emotional disturbances with family psychosocial problems, no evidence for anatomic or neuropathic bladder disease, and a spectrum of structural uropathology with bladder trabeculation, diverticula, reflux, and hydronephrosis. Urodynamic investigation typically reveals a large postvoid residual urine volume and individualized patterns of bladder-sphincter incoordination during voiding.

Attention has been called to the hazards of attempting surgical correction of reflux and sus- pected obstruction in patients with unrecognized and untreated detrusor-sphincter dyssynergia. ${ }^{18,25}$ Unfortunately, in the past the diagnosis was made too often only after operative failure. Treatment of the sequelae of this disordered voiding state depends first on an accurate urodynamic diagnosis, and then on restoring and normalizing bladder and sphincter activities. Success has been reported using a wide variety of conventional urologic techniques and unconventional methods ${ }^{18-25}$ which include: intermittent catheterization, timed voidings, bladder retraining, pharmacotherapy, biofeedback techniques, and hypnosis. The ultimate goal with any of these techniques is an individually tailored program aimed at the patient's specific voiding disturbances.

Pathophysiologically severe detrusorsphincter dyssynergia as occurs in the syndrome of non-neurogenic, neurogenic bladder is fortunately an uncommon entity, and even at large centers with a particular interest in the problem, relatively few of these cases have been collected. More common are the many neurologically normal children with less severe symptoms of voiding dysfunction and minimal or absent structural pathology. In most instances, these lesser disturbances do not require treatment, do not tend to progress, and are usually transient, reflecting the peculiar but probably normal ways in which children eventually develop urinary control.

University of Michigan Medical Center University Hospital, Box 03 Ann Arbor, Michigan 48109

\section{References}

1. Nash DFE: The development of micturition control with special reference to enuresis, Ann. R. Coll. Surg Engl. 5: 318 (1949).

2. Muellner SR: Development of urinary control in children, JAMA 172: 1256 (1960).

3. Diokno AC, Koff SA, and Bender LF: Periurethral striated muscle activity in neurogenic bladder dysfunction, J. U rol. 114: 743 (1974).

4. Koff SA, Lapides J, and Piazza DH: The uninhibited bladder in children: a cause for urinary obstruction, infection and reflux, in, Hodson J, and Kincaid-Smith P (Eds): Reflux Nephropathy, New York, Masson, 1979, p. 161.

5. Allen TD, and Bright TC: Urodynamic patterns in children with dysfunctional voiding problems, J. Urol. 119: 247 (1978).

6. Hinman F Jr: Syndromes of vesical incoordination, Urol. Clin. North Am. 7: 311 (1980).

7. Bauer SB, et al: The unstable bladder of childhood, ibid. 7: 321 (1980).

8. Johnston JH, Koff SA, and Glassberg KI. The pseudoobstructed bladder in enuretic children, Br. J Urol, 50: 505 (1978).

9. Koff SA, Lapides J, and Piazza DH: Association of urinary 
tract infection and reflux with uninhibited bladder contractions and voluntary sphincteric obstruction, J. Urol. 122: 373 (1979).

10. Lapides J, and Diokno AC: Persistence of the infant bladder as a cause for urinary infection in girls, Trans. Am. Assoc. Genitourin. Surg. 61: 51 (1969).

11. Van Gool JD: Bladder infection and pressure, p. 181, op cit. ${ }^{4}$

12. Vincent SA: Postural control of urinary incontinence. The curtsey sign, Lancet 2: 631 (1966).

13. Tanagho EA, Miller ER, Lyon RP, and Fisher R: Spastic striated external sphincter and urinary infection in girls, $\mathrm{Br}$. $\mathrm{J}$. Urol. 43: 69 (1971).

14. Tanagho EA, and Miller ER: Abnormal voiding and urinary tract infection, Int. Urol. Nephrol. 4: 165 (1972).

15. Van Gool J, and Tanagho EA: External sphincter activity and recurrent urinary tract infection in girls, Urology 10: 348 (1977).

16. Lapides J, and Costello RT Jr: Uninhibited neurogenic bladder: a common cause for recurrent urinary tract infection in normal women, J. Urol. 101: 539 (1969).
17. Diokno AC, and Lapides J: Oxybutynin: a new drug with analgesic and anticholinergic properties, ibid. 108: 307 (1972).

18. Hinman F Jr, and Bauman FW: Complications of vesicoureteral operations from incoordination of micturition, ibid. 116: 638 (1976).

19. IDEM: Vesical and ureteral damage from voiding dysfunction in boys without neurologic or obstructive disease, ibid. 109: $727(1973)$

20. Mix LW: Occult neuropathic bladder, Urology 10: 1 (1977).

21. Hinman F: Urinary tract damage in children who wet, Pediatrics 54: 142 (1974).

22. Allen TD: The non-neurogenic, neurogenic bladder, J. Urol. 117: 232 (1977).

23. Dorfman LE, Bailey J, and Smith JP: Subclinical neurogenic bladder in children, ibid. 101: 48 (1969).

24. Allen TD: Commentary on dysfunctional abnormalities of the urinary tract, Urol. Clin. North Am, 7: 357 (1980)

25. IDEM: Vesicoureteral reflux as a manifestation of dysfunctional voiding, p. 171 , op cit. ${ }^{4}$ 